\title{
Aircraft AC Generators: Hybrid System Modeling and Simulation
}

\author{
Ashraf Tantawy, Student Member, IEEE, Xenofon Koutsoukos, Senior Member, IEEE, \\ and Gautam Biswas, Senior Member, IEEE
}

\begin{abstract}
Integrated Drive Generators (IDGs) are the main source of electrical power for a number of critical systems in aircraft. Fast and accurate fault detection and isolation is a necessary component for safe and reliable operation of the IDG and the aircraft. IDGs are complex systems, and a majority of the existing fault detection and isolation techniques are based on signal analysis and heuristic methods derived from experience. Model-based fault diagnosis techniques are hypothesized to be more general and powerful in designing detection and isolation schemes, but building sufficiently accurate models of complex IDGs is a difficult task. dq0 models have been developed for design and control of generators, but these models are not suitable for fault situations, where the generator may become unbalanced. In this paper, we present a hybrid phase-domain model for the aircraft generator that accurately represents both nominal and parametric faulty behaviors. We present the details of the hybrid modeling approach and simulation results of nominal operation and fault behaviors associated with parametric faults in the aircraft generator. The simulation results show that the developed model is capable of accurately capturing the generator dynamics under a variety of normal and faulty configurations.
\end{abstract}

Index Terms-Hybrid modeling, integrated drive generator, phase-domain model, synchronous AC generator.

\section{INTRODUCTION}

The Integrated Drive Generator (IDG) is the primary source for electrical power in aircraft. The system draws its power from the main engines of the aircraft and comprises a number of subsystems that convert the mechanical energy into AC voltage. Fast and accurate fault detection and isolation is a necessary component for safe and reliable operation of the IDG and the aircraft. The majority of the existing techniques for fault detection and isolation of synchronous generators are model-free [3], [4], [11], [14]. On the other hand, model-based fault diagnosis techniques that utilize structural and analytic information contained in the system model are hypothesized to be more general and powerful in designing detection and isolation schemes [5], [6], [8]. However, building sufficiently accurate models of the IDG electrical subsystem is a difficult task because of the complex nonlinearities and the timevarying spatial relations involved in defining the dynamics of the electromagnetic behavior. In addition, the rectifier subsystem that converts the exciter AC voltage output into DC voltage that excites the main generator field includes switching components that introduce discrete dynamics into the overall system behavior.

The authors are with the Institute for Software Integrated Systems and the Department of Electrical Engineering and Computer Science, Vanderbilt University, Nashville, TN, 37235, USA (email: ashraf.tantawy@ vanderbilt.edu; xenofon.koutsoukos@vanderbilt.edu; gautam.biswas@vanderbilt.edu).
The traditional way of analyzing switching circuits relies on averaging or discretization techniques to make analysis of the circuit more tractable [15]. In this paper, we employ hybrid modeling [2], which combines the use of discrete and continuous behaviors to develop a hybrid model for the brushless AC generator that accurately captures the system dynamics. The model is used to simulate the machine behavior under both normal and faulty conditions. We explicitly model the exciter and main generators using the phase-domain representation, rather than the dq0 domain representation. dq0 modeling is useful for designing and developing controllers for nominal generator behavior [1], [9], while phase domain representations facilitate the simulation of a wide variety of transient phenomena in the machine, and this permits the generator to be employed in a variety of different load configurations.

Unlike other simulation work where the generator is part of a power distribution grid [1], [9], [13], we do not assume that the machine is connected to an infinite bus that is kept at constant balanced 3-phase voltage by other machines. The infinite bus assumption does not apply to the aircraft power distribution system, where the main generator output voltage can vary with input and load configuration changes. We do not assume that the output from the rectifier circuit is a constant DC voltage, but that it is determined by the dynamics of the modeled feedback loop. Also, it is well known that even under normal operating conditions, the rectifier output has ripples, and this can affect the transient behavior of the main generator, especially under fault conditions. We believe that the more detailed models will provide a framework for developing robust model-based diagnosis schemes that exploit analytic redundancy, and potentially reduce the number of sensors required for detection and isolation of faults and degradations in the system.

The rest of this paper is organized as follows: Section II summarizes existing approaches for fault detection and diagnosis of synchronous generators, emphasizing the need for exploration of model-based techniques. Section III describes the hybrid model for the synchronous generator part of the IDG. Section IV provides a classification of faults typically found in brushless AC generators. A subset of these faults are injected in the developed model to simulate the faulty system behavior. Section V summarizes simulation results for both normal and faulty behaviors. Finally Section VI concludes the work with future directions to extend the developed model. 


\section{RELATED WORK}

In this section, we review existing techniques to detect and isolate faults in brushless AC generators. Existing approaches are classified into three main categories: harmonic analysis, artificial neural networks, and model-based approaches.

\section{A. Harmonic Analysis}

A fault in the generator (inter-turn fault or ground fault) produces additional flux harmonics in the air gap that are related to the nature of the fault. Although the flux is not a pure sinusoidal even in normal operation (due to winding distribution), the additional harmonics under normal operating conditions are small and can be neglected. Under faulty conditions, the flux harmonics in the air gap increase in magnitude and produce noticeable harmonic currents and voltages in different windings. Different techniques are proposed to exploit these harmonics: harmonics induced in the field and armature windings during a fault condition are used to diagnose field and armature inter-turn faults, short-circuited diodes, phase to ground faults, and external faults [3], [16]. The harmonic current generated in the rotor winding is used to diagnose field and armature shorts [14]. Third harmonic voltages induced in the armature are widely used to detect ground faults [4]. However, third harmonic signals depend on generator construction and excitation, and change with varying loads, making the discrimination between ground faults and load fluctuations difficult.

\section{B. Artificial Neural Networks, ANN}

The power of neural networks to approximate functions with arbitrary complexity has been exploited in a number of ways. In [11], a neural network is trained using various data sets, with field and armature currents as inputs and one of three states: normal, internal fault, or external fault as output. Previous sample values are used as inputs to train the neural network on the dynamic behavior of the machine. A second neural network is used to further classify the faulty phase in case of an internal fault.

The difficulty with using neural networks is that they require large, comprehensive data sets that capture different modes of operation of the machine and a number of different fault conditions and magnitudes. Different combinations of loads, faults, fault locations and magnitudes, and current values are required to generate the training data sets. Unless sufficient data is available to cover a majority of these conditions the neural network classifier will be incomplete and it may generate incorrect results.

\section{Model-based FDI}

A model-based diagnosis approach is found in [17]. The excitation current can be calculated by the mathematical model and compared with the measured exciting current. Rotor winding inter-turn faults are diagnosed by this method. In this paper we explore this approach further by presenting an accurate hybrid model that captures machine transients.

\section{Brushless AC Generator Model}

In this paper, we focus on the electrical subsystems of the IDG, and assume that the mechanical subsystem provides a constant angular velocity. Figure 1 shows the block diagram for the primary electrical subsystems of the IDG. The main generator is connected directly to aircraft loads (via line contactors). To keep the terminal voltage at or close to its operating value, a feedback loop is employed. It includes the Generator Control Unit (GCU) that compares the measured terminal voltage to a reference value, and regulates the field voltage for the exciter generator. The 3 phase output voltage of the exciter generator is then rectified and the DC voltage signal (with ripples) produced by the rectifier is applied to the field winding of the main generator. A Permanent Magnet Generator (not shown) is used as a power source for the GCU.

The armature of the exciter generator, rectifier, and the field of the main generator (enclosed in a gray dashed rectangular box) rotate with the main shaft that drives the entire IDG system. The rest of the electrical subsystem (exciter field, main generator armature, GCU, and the load) are stationary (a scheme that is widely known as brushless generator). The block diagram numbers correspond to the order in which the details of the subsystem models are presented in the following subsections. The first subsystem includes the exciter generator and the rectifier and is modeled as a hybrid system (Section III-A). The second subsystem is the main generator with load and is modeled as a continuous time-varying system (Section III-B). For this paper the third subsystem, the GCU, is modeled as a simple PI controller.

To simplify the notation, we use the same parameter names and variables for both the exciter generator and main generator. This should not be confusing since each generator is modeled separately and described in a separate section. In practice, the parameter values may or may not be the same. A glossary of variables is listed in Table I, where subscript $x \in\{a, b, c, k q, f d, k d\}$ represents the different generator windings: $a, b$ and $c$ are the 3-phase windings, $k q$ and $k d$ are the two damper windings, and $f d$ is the field winding.

TABLE I

Glossary of Model Variables

\begin{tabular}{|l||l|}
\hline Variable & Description \\
\hline$i_{x}$ & Winding-x current. \\
\hline$v_{x}$ & Winding-x voltage. \\
\hline$\lambda_{x}$ & Winding-x flux linkage. \\
\hline$r_{x}$ & Winding-x resistance. \\
\hline$\omega$ & Angular velocity. \\
\hline$\theta_{r}$ & Angular displacement. \\
\hline \hline
\end{tabular}

\section{A. Hybrid Model for the Exciter and Rectifier}

Figure 2 shows the electrical connections between the exciter generator and the full wave diode rectifier. The output voltage, across a resistive load is connected to the field windings of the main generator.

The modes of operation for the exciter-rectifier are defined by the forward and reverse-biased diodes. Diodes are modeled as ideal switches, so they act as a short circuit when forward 

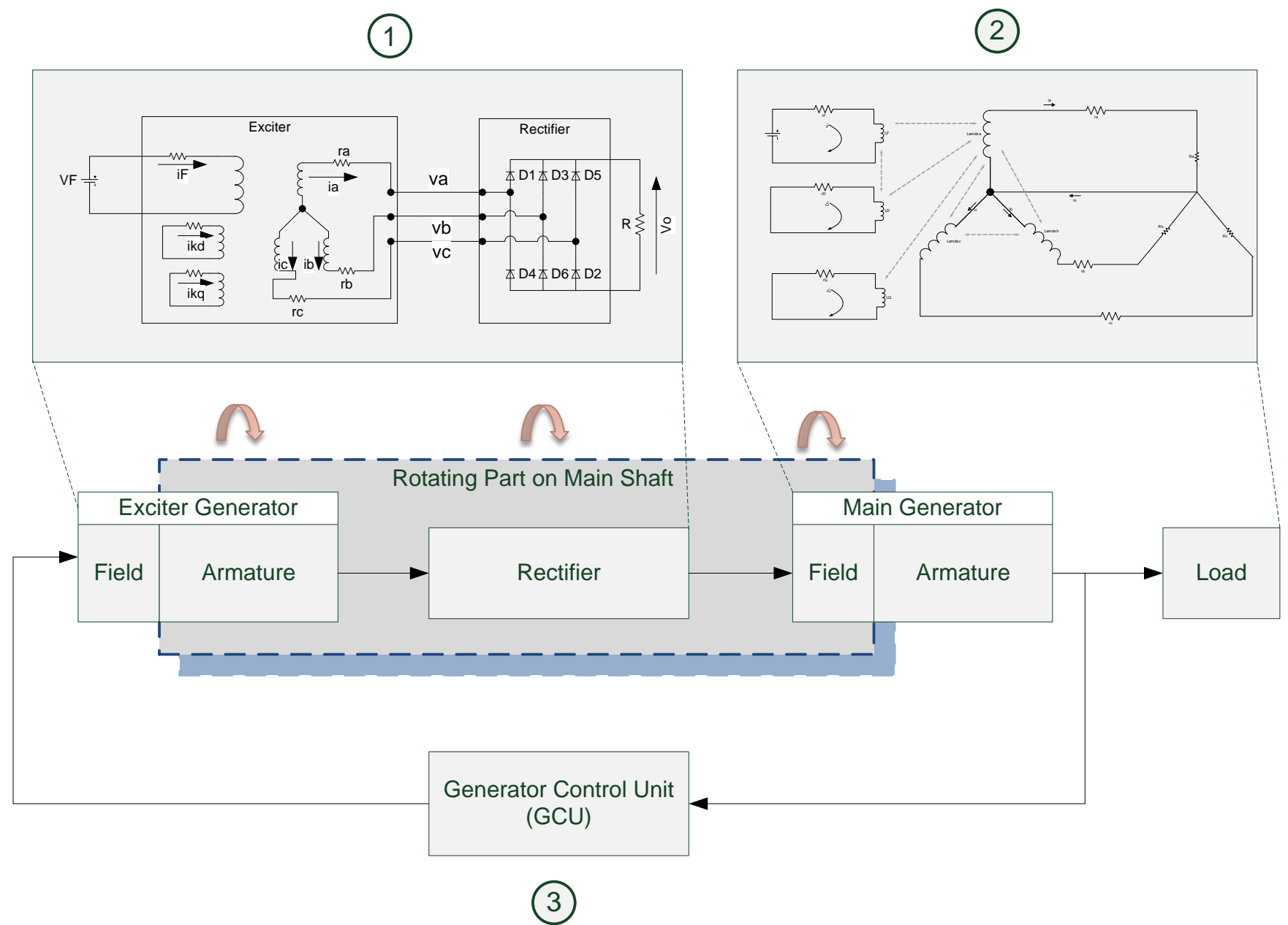

Fig. 1. IDG electrical subsystem block diagram

biased, and open circuit when reverse biased. As an example, when $V_{a} \geq V_{b}$ and $V_{b} \geq V_{c}$ diodes D1 and D6 are forwardbiased and all other diodes are reverse-biased. We call that mode AB (first letter specifies the largest voltage and last letter specifies the smallest voltage). Similarly we can define the 6 modes of operation for the exciter-rectifier:

1) Mode AB: diodes D1 and D6 are forward biased, and all other diodes are reverse biased.

2) Mode AC: diodes D1 and D2 are forward biased, and all other diodes are reverse biased.

3) Mode BC: diodes D3 and D2 are forward biased, and all other diodes are reverse biased.

4) Mode BA: diodes D3 and D4 are forward biased, and all other diodes are reverse biased.

5) Mode CA: diodes D5 and D4 are forward biased, and all other diodes are reverse biased.

6) Mode CB: diodes D5 and D6 are forward biased, and all other diodes are reverse biased.
The 6 modes of operation define the hybrid model for the exciter-rectifier system [2].

1) Discrete Dynamics: Figure 3 shows the system automaton with the six discrete modes defined above. The system switches from one mode to another based on the terminal voltages of the exciter generator. The guard conditions for the automaton describing the mode transitions are shown in Figure 3.

2) Continuous Dynamics: We present here continuous dynamics for one mode only (mode $\mathrm{AB}$ ). The dynamics for the remaining discrete states can be derived in a similar way.

In Mode $\mathrm{AB}$, windings $a$ and $b$ are connected together and winding $c$ is floating (see Figure 3). Therefore,

$$
\begin{gathered}
\dot{i_{a}}+\dot{i_{b}}=0 \\
\dot{i_{c}}=0
\end{gathered}
$$

It is possible to reduce the number of state variables by 2 , but we represent the system in terms of 7 state variables (6 winding currents and the angular displacement). This keeps the 


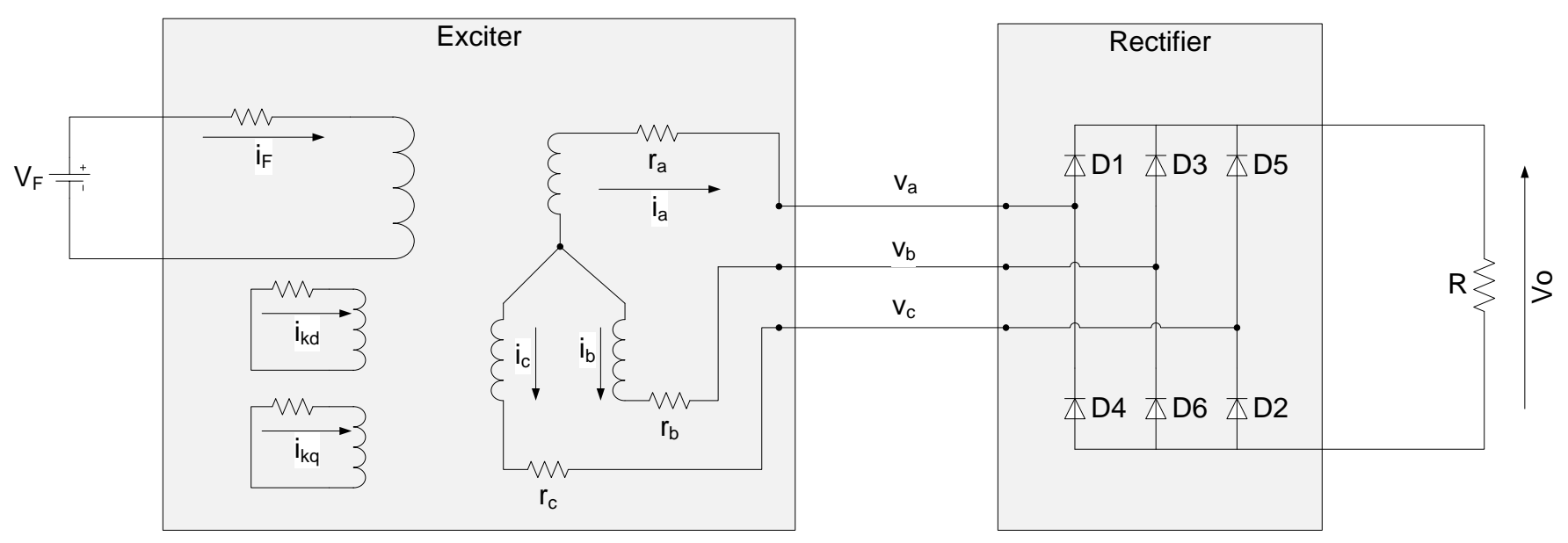

Fig. 2. Exciter and Rectifier Electrical Schematic Diagram

model representation consistent between the different modes (in terms of the state variables).

In addition to equations (1) and (2), the following equation is derived from the loop that includes phase $a$ and phase $b$ :

$$
\dot{\lambda_{a}}-\dot{\lambda_{b}}=\left(r_{a}+r_{b}+R\right) i_{a}
$$

The field winding and damper windings voltage equations are expressed as:

$$
\left[\begin{array}{c}
0 \\
V_{F} \\
0
\end{array}\right]=\left[\begin{array}{ccc}
r_{k q} & 0 & 0 \\
0 & r_{f d} & 0 \\
0 & 0 & r_{k d}
\end{array}\right]\left[\begin{array}{c}
i_{k q} \\
i_{f d} \\
i_{k d}
\end{array}\right]+\left[\begin{array}{c}
\dot{\lambda_{k q}} \\
\dot{\lambda_{f d}} \\
\dot{\lambda_{k d}}
\end{array}\right]
$$

The flux linkages-currents relationship is expressed as:

$$
\lambda=\mathbf{L i}
$$

where:

$$
\begin{gathered}
\lambda=\left[\begin{array}{llllll}
\lambda_{a} & \lambda_{b} & \lambda_{c} & \lambda_{k q} & \lambda_{f d} & \lambda_{k d}
\end{array}\right]^{T} \\
\mathbf{i}=\left[\begin{array}{llllll}
i_{a} & i_{b} & i_{c} & i_{k q} & i_{f d} & i_{k d}
\end{array}\right]^{T} \\
\mathbf{L}=\left[\begin{array}{cc}
-L_{s} & L_{s r} \\
-L_{s r}^{T} & L_{r}
\end{array}\right]
\end{gathered}
$$

$L_{s}, L_{s r}, L_{r}$ are given by equations (12)-(14) in the appendix. Using equations (1)-(6), we get the following state space model:

$$
\frac{d \mathbf{i}}{d t}=\mathbf{M}^{-\mathbf{1}}(\mathbf{V}+\mathbf{N i})
$$

where:

$$
\mathbf{V}=\left[\begin{array}{llllll}
0 & 0 & 0 & 0 & V_{F} & 0
\end{array}\right]^{T}
$$

The expression for $\mathbf{M}$ and $\mathbf{N}$ are given by equations (15)(20) listed in the appendix. We assume that the angular velocity is held constant, and the remaining dynamic equation is for the rotor angle:

$$
\dot{\theta_{r}}=\omega
$$

The terminal voltages for the exciter are given by the vector equation:

$$
\mathbf{V}_{\mathbf{a b c}}=\lambda_{\mathbf{a b c}}^{\cdot}-\mathbf{r}_{\mathbf{a b c}} \dot{\mathbf{i}}_{\mathbf{a b c}}
$$

The terminal voltages define the current discrete mode of the system. The output voltage from the system is taken across the load resistance $R$, and depends on the discrete state. Both terminal voltages and output voltage are calculated as part of the dynamics defined for every discrete state.

To summarize the hybrid model (Figure 3 ) we have six modes $\{A B, A C, B C, B A, C A, C B\}$ defined by the combinations of on and off diodes. With every mode there is an associated continuous dynamic model (equation (7)), with state variables $X=\left\{i_{a}, i_{b}, i_{c}, i_{k q}, i_{f d}, i_{k d}, \theta_{r}\right\}$. The initial condition for the system is assumed to be mode $A B$ and all state variables are set to zero. The transition from one mode to another is defined by the exciter generator terminal voltages (equation (9)). For example, in mode $A B$ the transition to mode $A C$ occurs only when the condition $V_{b}>V_{c}$ is satisfied. When the system makes a transition from one mode to another, a reset condition is applied to the state variables. For example, when entering mode $A B$ the reset condition is given by $\left(i_{c}=0, i_{a}=-i_{b}\right)$.

\section{B. Main Generator Model}

The state equations governing the behavior of the main generator system are derived from Figure 4 where:

$$
\dot{\lambda}=\mathbf{r i}+\mathbf{v}
$$

$$
\mathbf{v}=\left[\begin{array}{llllll}
v_{a} & v_{b} & v_{c} & 0 & V_{F} & 0
\end{array}\right]^{T}
$$




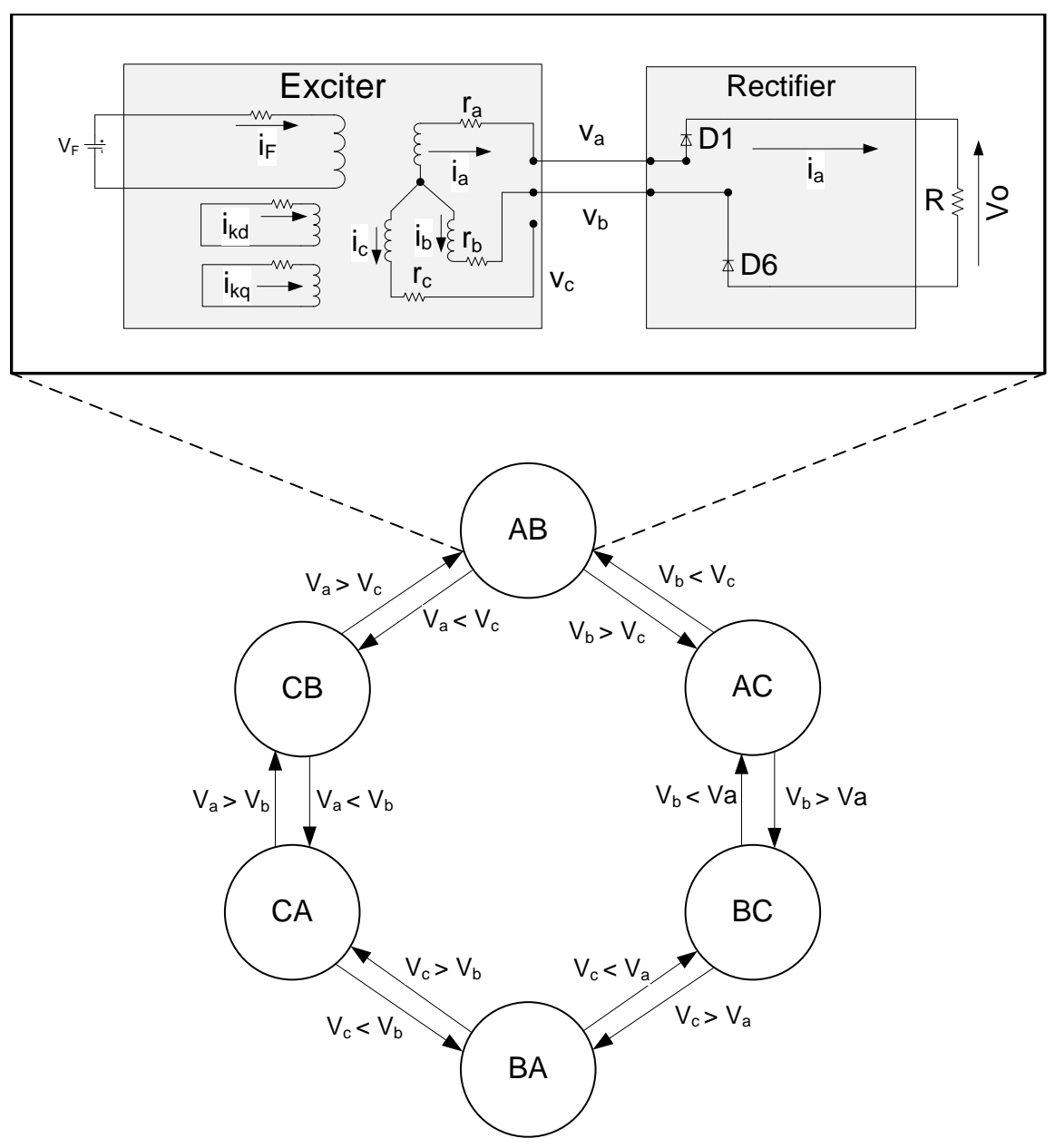

Fig. 3. System Automaton

$$
\mathbf{r}=\operatorname{diag}\left(\begin{array}{cccccc}
r_{a} & r_{b} & r_{c} & -r_{k q} & -r_{f d} & -r_{k d}
\end{array}\right)
$$

From equations (5), (6), and (10) we obtain the state space model for the synchronous generator:

$$
\dot{\lambda}=\mathbf{r} \mathbf{L}^{-1} \lambda+\mathbf{v}
$$

The state variables were chosen as the flux linkages. It is common also to take winding currents as state variables. Equation (11) with the load equations completely specify the system for simulation.

\section{Generator Control Unit (GCU)}

The GCU is modeled as a simple PI controller. The input to the GCU (the rms value for the main generator 3-phase voltages) is compared to a reference value, and the error signal is the input to the PI controller. The PI controller output determines the exciter generator field voltage. For the simulated model parameters the PI controller is tuned to a proportional gain $K_{p}=3$, and an integral gain $K_{i}=5$.

\section{AC Generator Fault Classification}

In this section we present a classification of faults that are typically encountered during operation of AC generators. A subset of this list will be used in Section $\mathrm{V}$ to inject faults into the developed model to study the resultant faulty behaviors.

Generator faults can be classified into one of two main categories: parametric faults and structural faults. Parametric faults are characterized by a change in the magnitude of one (or more) system model parameters. These faults do not affect the structure of the system, and, therefore, the system model is still a valid representation for the actual system. Structural faults change the system configuration and cannot be represented by a magnitude change in the system parameters. The system model under these faults is no longer valid, and a new model representing the new configuration is necessary to generate the system behavior.

Structural faults can be classified into internal faults and external faults. External faults are the ones that happen outside the machine terminals, and although they do not change the structure of the machine, the overall system model changes. Sometimes it is possible to represent external faults by a parameter change by introducing auxiliary elements in the original model, but this may not always be possible. Internal 


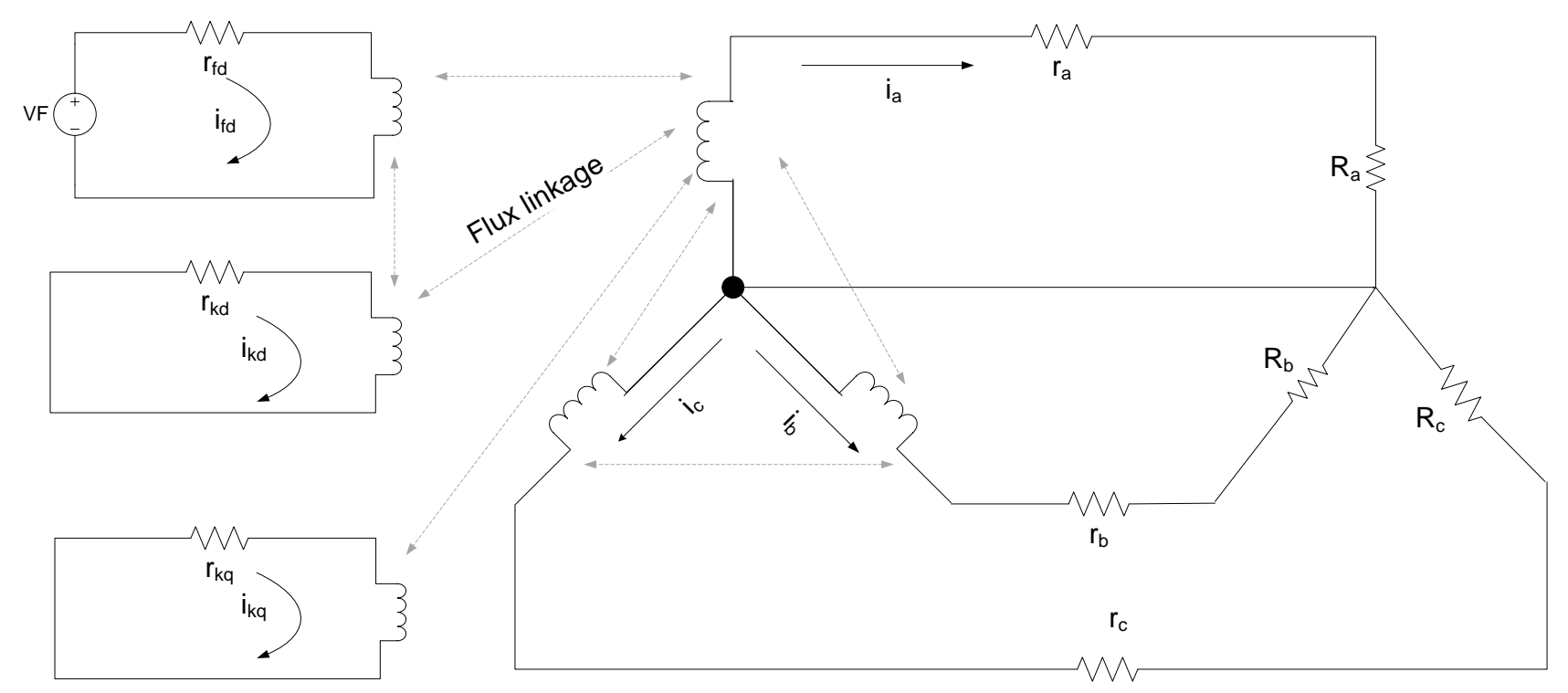

Fig. 4. Main generator schematic diagram

faults are intrinsic to the machine itself (within the machine boundary).

In some situations, parametric and structural faults are not independent. For example, an incipient parametric fault representing a small number of shorted turns may cause overheating in the magnetic core of the generator, which after a period of time causes structural winding to ground fault. Therefore, detecting incipient faults at early stages is very important for machine protection.

\section{A. Parametric Faults}

Following the model presented in Section III, the physical parameters of the synchronous generator are summarized in Table II. All other model parameters (inductances) can be expressed as a function of one or more of the parameters in Table II.

One should note that this list of parameters is valid only under nominal operations with no faults. This is because the stator windings are assumed symmetric, and, therefore, the number of turns for all windings are represented by a single parameter $\left(N_{s}\right)$. Similarly, the mutual inductance between every stator winding and the other machine windings (field and damper windings) is represented by a single parameter; for example $L_{s f d}$. Under faulty conditions, there should be a distinct representation for the faulty winding by using different parameters. As an example, given a fault in winding $a$, the new parameters that need to be introduced into the model are listed in Table III. Since $r, l, \alpha_{1}$, and $\alpha_{2}$ represent the geometry for the machine, they are not considered as fault candidates.

\section{B. Structural Faults}

1) External Faults: Phase to ground and phase to phase short circuits are the most common external faults.
TABLE II

SYNCHRONOUS GENERATOR PHYSICAL PARAMETERS

\begin{tabular}{|c||l|}
\hline \multicolumn{1}{|c||}{ Parameter } & $\begin{array}{l}\text { Description } \\
\text { Radius to the mean of the air gap (to the inside } \\
\text { circumference of the stator }\end{array}$ \\
\hline$\alpha_{1}, \alpha_{2}$ & Axial length of the air gap of the machine \\
\hline$N_{s}$ & $\begin{array}{l}\text { Neometrical constants } \\
\text { tributed stator winding, which would give rise to the } \\
\text { same fundamental component as the actual winding } \\
\text { distribution. }\end{array}$ \\
\hline$N_{f d}$ & $\begin{array}{l}\text { Number of turns of the equivalent sinusoidally dis- } \\
\text { tributed field winding. }\end{array}$ \\
\hline$N_{k q}$ & $\begin{array}{l}\text { Number of turns of the equivalent sinusoidally dis- } \\
\text { tributed damper winding on q-axis. }\end{array}$ \\
\hline$N_{k d}$ & $\begin{array}{l}\text { Number of turns of the equivalent sinusoidally dis- } \\
\text { tributed damper winding on d-axis. }\end{array}$ \\
\hline$L_{l s}$ & Stator winding leakage inductance. \\
\hline$L_{l f d}$ & Field winding leakage inductance. \\
\hline$L_{l k q}$ & Damper winding q leakage inductance. \\
\hline$L_{l k d}$ & Damper winding d leakage inductance. \\
\hline$r_{a}$ & Stator winding a resistance. \\
\hline$r_{b}$ & Stator winding b resistance. \\
\hline$r_{c}$ & Stator winding c resistance. \\
\hline$r_{f d}$ & Field winding resistance. \\
\hline$r_{k q}$ & Damper winding q resistance. \\
\hline$r_{k d}$ & Damper winding d resistance. \\
\hline \hline
\end{tabular}

2) Internal Faults: An example of an internal fault that changes the structure of the system is one phase to ground fault. The short circuit from winding to the ground creates a new loop with additional current component and with loop voltage equal to 0 [12]. The situation becomes more complex when there is a dual fault from two phases to the ground.

\section{Simulation Results}

The complete AC generator model is simulated in Matlab ${ }^{\circledR /}$ Simulink $^{\circledR}$ [10] with a balanced resistive load $(100 \Omega)$ and a 
TABLE III

NEW SYNCHRONOUS GENERATOR PARAMETERS (WINDING-A FAULT)

\begin{tabular}{|c||l|}
\hline Parameter & Description \\
\hline$N_{s a}$ & Equivalent number of turns in the winding a. \\
\hline$L_{\text {safd }}$ & $\begin{array}{l}\text { Mutual inductance between winding a and field wind- } \\
\text { ing. }\end{array}$ \\
\hline$L_{\text {sakd }}$ & $\begin{array}{l}\text { Mutual inductance between winding a and damper } \\
\text { winding kd. }\end{array}$ \\
\hline$L_{\text {sakq }}$ & $\begin{array}{l}\text { Mutual inductance between winding a and damper } \\
\text { winding kq. }\end{array}$ \\
\hline$L_{\text {sas }}$ & $\begin{array}{l}\text { Mutual inductance between winding a and winding b } \\
\text { (or c). }\end{array}$ \\
\hline \hline
\end{tabular}

PI controller to regulate the main generator output voltage at $115 \mathrm{~V}$ RMS. A parallel capacitor is added at the rectifier output instead of the resistance to help smoothing the voltage applied to the main generator field. A capacitance value of 1 Farad is used in the simulation.

Table IV shows a set of parameter values (in SI units) for a built in synchronous machine in Simulink ${ }^{\circledR}$, which is in dq0 domain. For the simulated model these values are transformed to the phase-domain values using the equations shown in Table $\mathrm{V}$. The transformation is not unique since more than one ratio of the windings may result in the same set of parameter values in phase domain.

TABLE IV

DQ0 MODEL PARAMETERS

\begin{tabular}{|l|l||l|l|}
\hline Parameter & Value & Parameter & Value \\
\hline$L_{l s}$ & $0.004527 \mathrm{H}$ & $L_{m d}$ & $0.1086 \mathrm{H}$ \\
\hline$L_{m q}$ & $0.05175 \mathrm{H}$ & $\hat{L}_{l k q}$ & $0.01015 \mathrm{H}$ \\
\hline$\hat{L}_{l f d}$ & $0.01132 \mathrm{H}$ & $\hat{L}_{l k d}$ & $0.007334 \mathrm{H}$ \\
\hline$r_{a}$ & $1.62 \Omega$ & $r_{b}$ & $1.62 \Omega$ \\
\hline$r_{c}$ & $1.62 \Omega$ & $\hat{r}_{k q}$ & $4.772 \Omega$ \\
\hline$\hat{r}_{f d}$ & $1.208 \Omega$ & $\hat{r}_{k d}$ & $3.142 \Omega$ \\
\hline$N_{s}$ & 100 & $N_{f d}$ & 100 \\
\hline$N_{k q}$ & 100 & $N_{k d}$ & 100 \\
\hline \hline
\end{tabular}

\section{A. Normal Behavior}

The normal model is simulated for $2 \mathrm{sec}$ starting from zero initial conditions for all state variables and the following variables are plotted:

- Rectifier output voltage (Figure 5). It is clear that the steady state output is a DC voltage with very small ripples that are smoothed by the parallel capacitor at the rectifier output.

- Main generator 3-phase voltage (Figure 6). After the transient period the 3-phase output from the main generator is stabilized at $115 \mathrm{~V}$ RMS.

- Main generator state currents (Figure 7). This includes the 3-phase output currents from the generator, field current, and damper winding currents. Damper winding currents are non-zero during the transient period only and then go to zero. The field current approaches its steady state DC value after the transient period.

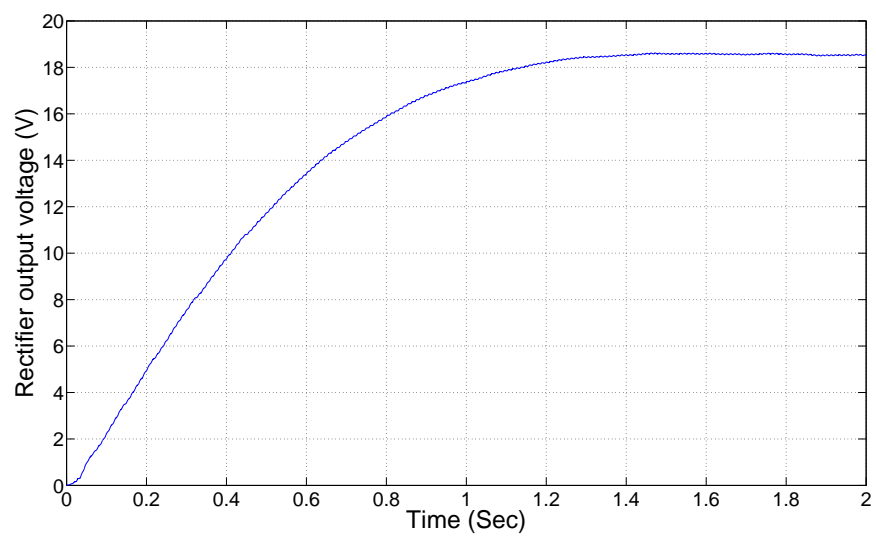

Fig. 5. Rectifier Output Voltage

TABLE V

Phase-domain Model Parameters Calculation

\begin{tabular}{|l|l||l|l|}
\hline Parameter & Expression & Parameter & Expression \\
\hline$L_{A}$ & $\frac{L m d+L m q}{3}$ & $L_{B}$ & $\frac{L m d-L m q}{3}$ \\
\hline$L_{l k q}$ & $\left(\frac{2}{3}\right)\left(\frac{N_{k q}}{N_{s}}\right)^{2} \dot{L}_{l k q}$ & $L_{m k q}$ & $\left(\frac{2}{3}\right)\left(\frac{N_{k q}}{N_{s}}\right)^{2} L_{m q}$ \\
\hline$L_{l f d}$ & $\left(\frac{2}{3}\right)\left(\frac{N_{f d}}{N_{s}}\right)^{2} \dot{L}_{l f d}$ & $L_{l k d}$ & $\left(\frac{2}{3}\right)\left(\frac{N_{k d}}{N_{s}}\right)^{2} \dot{L}_{l k d}$ \\
\hline$L_{s k q}$ & $\left(\frac{2}{3}\right)\left(\frac{N_{k q}}{N_{s}}\right) L_{m q}$ & $L_{s f d}$ & $\left(\frac{2}{3}\right)\left(\frac{N_{f d}}{N_{s}}\right) L_{m d}$ \\
\hline$L_{s k d}$ & $\left(\frac{2}{3}\right)\left(\frac{N_{k d}}{N_{s}}\right) L_{m d}$ & $L_{f d k d}$ & $\left(\frac{N_{f d}}{N_{k d}}\right) L_{m k d}$ \\
\hline$r_{k q}$ & $\left(\frac{2}{3}\right)\left(\frac{N_{k q}}{N_{s}}\right)^{2} r_{k q}$ & $r_{f d}$ & $\left(\frac{2}{3}\right)\left(\frac{N_{f d}}{N_{s}}\right)^{2} r_{f d}$ \\
\hline$r_{k d}$ & $\left(\frac{2}{3}\right)\left(\frac{N_{k d}}{N_{s}}\right)^{2} r_{k d}$ & & \\
\hline \hline
\end{tabular}

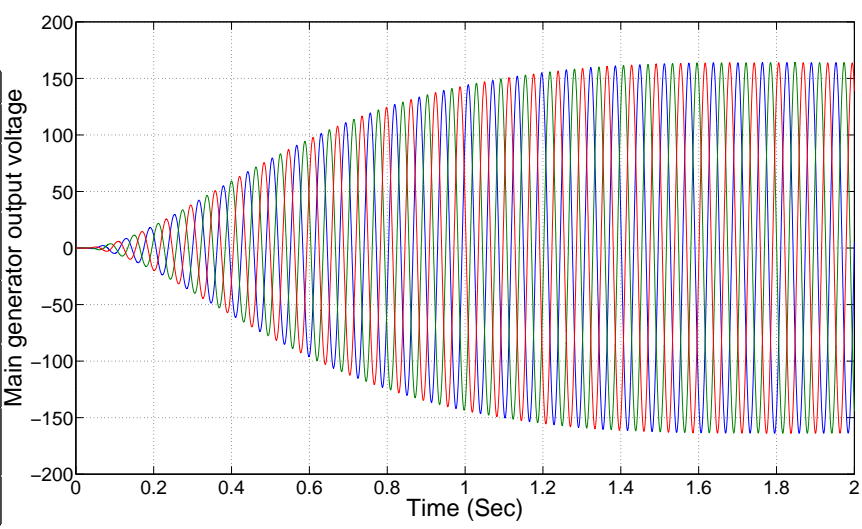

The implementation of the hybrid model presented in the paper is useful for general use and different simulation experiments since it is encapsulated in SimPower interface (SimPowerSystems $^{\text {TM }}$ software is a library of power engineering blocks that operates in Simulink ${ }^{\circledR}$ environment) [7]. Therefore, the developed model can be dragged and dropped in any network simulation.
Fig. 6. Main Generator Output Voltage

\section{B. Faulty Behaviors}

Following the fault classification presented in Section IV, only parametric faults are considered in this paper. The first fault to be considered is an abrupt jump in the resistance of the 

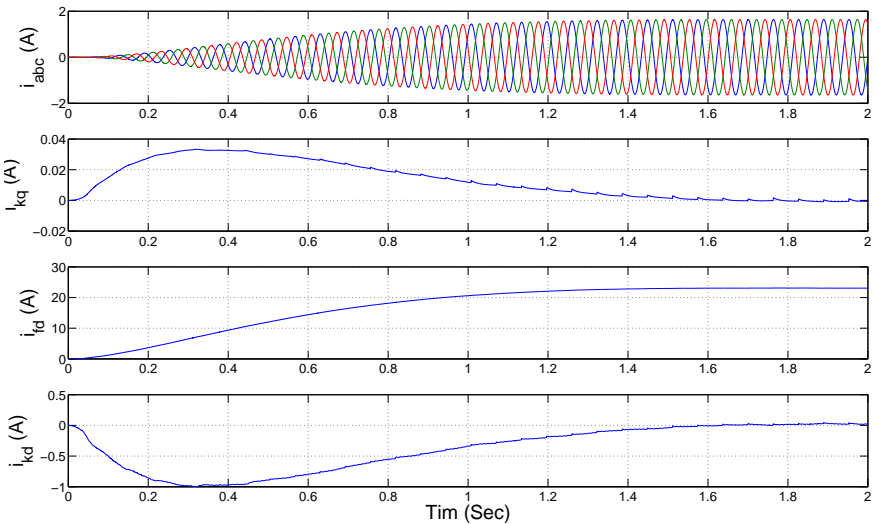

Fig. 7. Main Generator Winding Currents

field winding of the exciter generator. In practice, this may be caused by overheating of the winding. The nominal value for the resistance is $r_{f d}=0.8 \Omega$ and the faulty value is $r_{f d}=1 \Omega$. The fault is injected at simulation time $t=2 \mathrm{sec}$ and system variables are plotted:

- Rectifier output voltage (Figure 8). The voltage increases due to the existence of the controller to compensate for the new generator dynamics. It reaches a new steady state value after about $3 \mathrm{sec}$. The new value stabilizes the 3 phase output voltage for the main generator under a faulty condition at $115 \mathrm{~V}$ RMS.

- Main generator 3-phase voltage (Figure 9). The fault is evident by the sudden decrease of the amplitude of the three phase voltages. The closed loop controller brings the system to its operating condition within about $3 \mathrm{sec}$.

- Main generator state currents (Figure 10). For the 3phase currents (subplot 1) a sudden decrease occurs then the controller brings the current signals back to their nominal values. The damper winding $k q$ current (subplot 2) and field winding $f d$ current (subplot 3 ) undergo a negative spike, while damper winding $k d$ current (subplot 4) undergoes a positive spike. Note that it takes the field winding current more time to return to its normal operating value, while the damper winding currents return to zero much faster.

For the type of fault presented, it may be more appropriate to use the field winding current, since it has slower dynamics compared to damper winding currents. Also, phase currents have much higher frequency and to detect faults from these waveforms, either faster sensors are required or frequency response analysis is needed. However, one should note that the field and damper winding current measurements are not directly available since their relative windings are mounted on the rotating part of the generator and it is usually hard to install sensors for reliability issues. Observability of the AC generator presented here and state estimation is an interesting subject that needs to be addressed.

The second fault injected is a partial decrease in the effective number of turns of the exciter field windings $N_{f d}$. In practice this may be due to shorted turns. However, this fault cannot

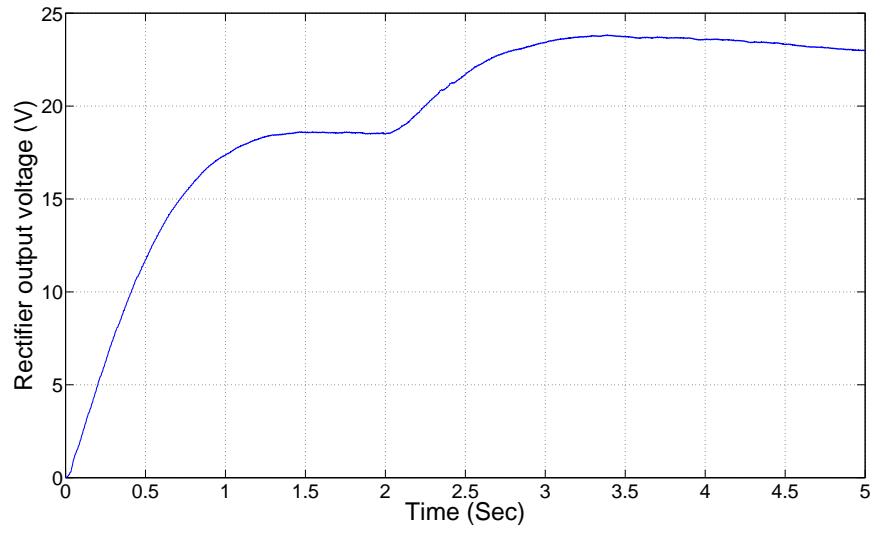

Fig. 8. Rectifier Output Voltage-Field winding resistance fault

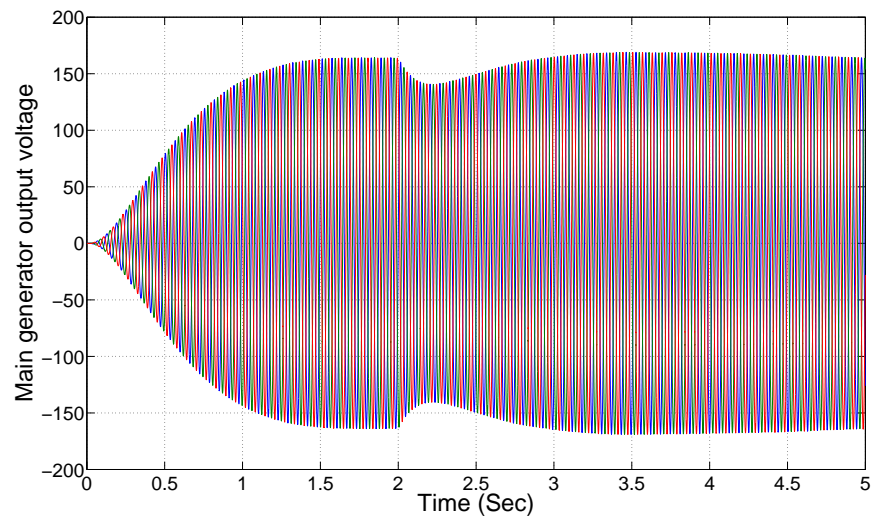

Fig. 9. Main Generator Output Voltage-Field winding resistance fault

be represented by a change in $N_{f d}$ only, since there are other parameters expressed as a function of $N_{f d}$. The list of these parameters is shown in Table VI.

It is assumed that $N_{f d}$ is decreased by $10 \%$. Other affected parameters are calculated according to the formulas presented in the appendix. The fault is injected at $t=2 \mathrm{sec}$ and system variables are plotted:

- Rectifier output voltage (Figure 11). The voltage decreases due to the existence of the closed loop controller to compensate for the new generator dynamics. It reaches a new steady state value after about $3 \mathrm{sec}$. The new value stabilizes the 3-phase output voltage for the main generator under a faulty condition at $115 \mathrm{~V}$ RMS.

- Main generator 3-phase voltage (Figure 12). The 3phase voltage is disturbed by a small amount due to this

TABLE VI

AFFECTED PARAMETERS BY A CHANGE IN FIELD WINDING TURNS

\begin{tabular}{|c||l|}
\hline Parameter & Description \\
\hline$L_{s f d}$ & $\begin{array}{l}\text { Mutual inductance between stator windings and field } \\
\text { winding. }\end{array}$ \\
\hline$L_{m f d}$ & Magnetizing inductance for the field winding. \\
\hline$L_{f d k d}$ & $\begin{array}{l}\text { Mutual inductance between damper winding kd and } \\
\text { field winding. }\end{array}$ \\
\hline$r_{f d}$ & Field winding resistance. \\
\hline \hline
\end{tabular}



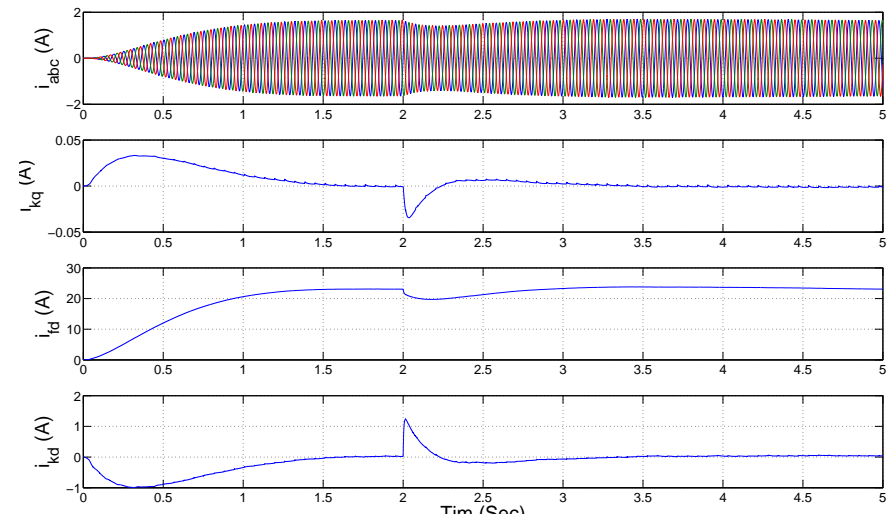

Fig. 10. Main Generator Winding Currents-Field winding resistance fault

fault, with small transient period. It then sets back to the regulated value of $115 \mathrm{~V}$ RMS. Due to the small transient period after fault injection and the existence of the controller in the loop, it may be hard to detect the fault by solely using the output voltage signals.

- Main generator state currents (Figure 13). A very similar effect is noticed in the current waveforms with one exception for the 3-phase currents. The magnitude of the change is very small and may not be even noticed. However, the change in damper winding and field winding current signals is significant during the transient period following fault injection. Damper winding currents return to their zero steady state value, while the field winding current sets to a new DC value to regulate the voltage under the new faulty conditions.

When performing fault detection in the time domain using the phase-domain representation for the machine, the 3-phase voltages and currents (which are usually the measurements) may not be the appropriate signals for the task. Faults could be detected easily by using other system variables like rectifier output voltage, damper winding currents, and main generator field current. However, as pointed out before, these variables are not measurable and state estimation may be needed.

The faults considered in this paper do not affect the capabilities of the feedback controller to bring the machine back to its regulated condition. It may happen, depending on the system new dynamics after the fault, that the tuned controller may not be able to regulate the machine. Fault adaptive control is required in this case to automatically tune the controller based on the new system dynamics. This is not addressed in the current study and it is of interest for further research.

\section{CONCLUSIONS}

In this paper we have presented a hybrid model for an aircraft brushless AC generator, modeled in phase-domain representation, which is more appropriate for asymmetrical and faulty machine analysis. The model was simulated successfully for both normal and parametric fault conditions, and the effect of faults on different system variables has been investigated. Simulation results have shown that faults are apparent in some

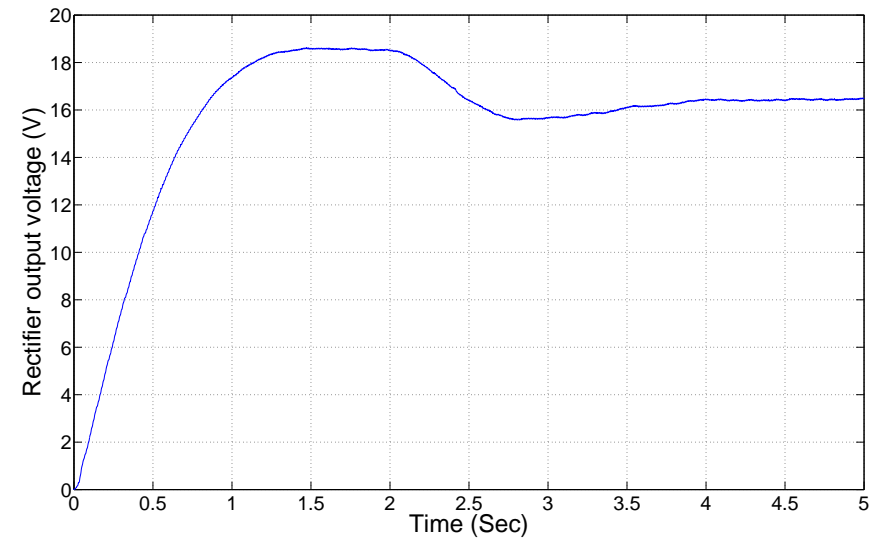

Fig. 11. Rectifier Output Voltage-Field winding turns fault

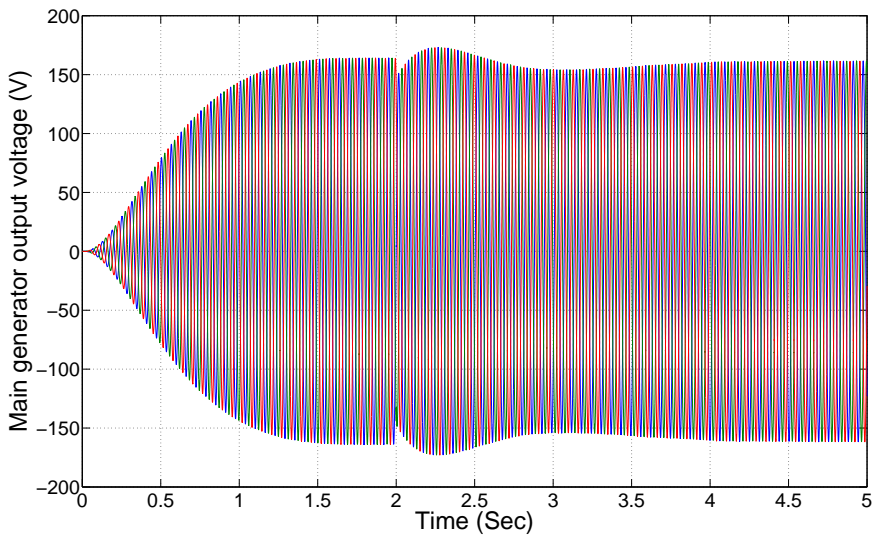

Fig. 12. Main Generator Output Voltage-Field winding turns fault

signals, like damper winding currents, more than machine terminal variables. However, damper winding currents are not directly measured and accurate estimation methods are required if these signals will be used for fault detection and isolation.

As future work, we will study the efficiency of using the developed model to detect and isolate faults assuming noisy measurements. The available measurements are terminal voltages and currents only, with no sensors on the rotating part of the machine. Hybrid system observability and state estimation will be studied to investigate the possibility of detecting and isolating faults. Also the hybrid model could be extended to incorporate other phenomena that are prevalent during faulty conditions like harmonics and magnetic saturation. These phenomena become especially important with structural faults. In this case we will have a hierarchical hybrid model where each mode represents either normal operation or a specific fault type.

Discrete faults in cables and connectors and their effect on the machine behavior will be studied. These faults are very important practically since they occur frequently during normal operation of the machine. Particular attention will be given to discrete faults in cables connecting terminal sensors to the GCU, since faults in these cables may cause delayed fault 

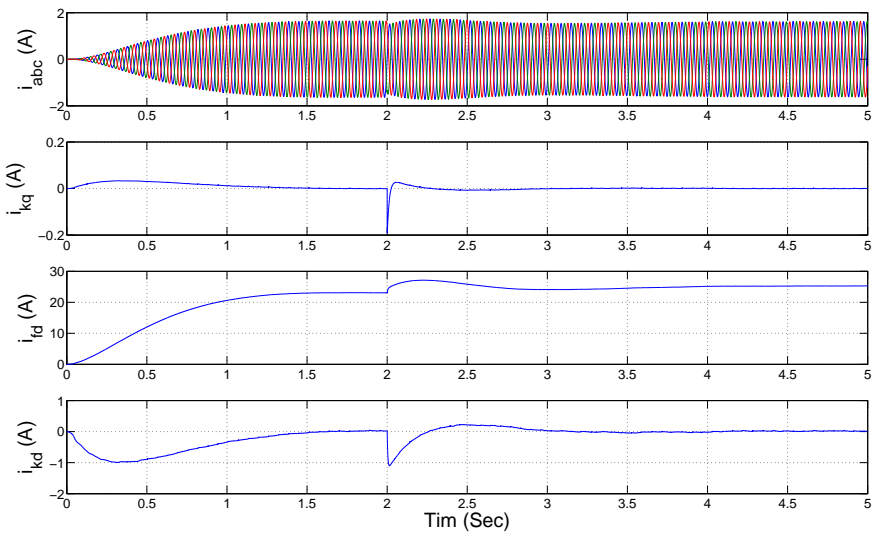

Fig. 13. Main Generator Winding Currents-Field winding turns fault

detection, misclassification of faults, or even loss of machine control.

\section{ACKNOWLEDGMENT}

This work was supported in part by the National Science Foundation under Grants CNS-0615214 and CNS-0347440, and NASA NRA NNX07AD12A.

\section{REFERENCES}

[1] P. M. Anderson and A. A. Fouad, Power System Control and Stability. Piscataway, NJ: IEEE Press Power Engineering Series, 2005.

[2] P. J. Antsaklis and X. D. Koutsoukos, "Hybrid systems: Review and recent progress," in Software-Enabled Control: Information Technology for Dynamical Systems. Wiley-IEEE, 2003.

[3] Z. Bo, M. Redfern, S. Potts, S. Weller, N. Chin, and F. Jiang, "Nondifferential protection of a generator's stator utilizing fault transients," IEE Seventh International Conference on Developments in Power System Protection, pp. 503-506, 2001

[4] W. Breingan, C. Castro, J. Latham, J. Mescua, A. Phadke, J. Postforoosh, E. Schweitzer, W. Strang, F. Tajaddodi, and E. Udren, "Survey of experience with generator protection and prospects for improvements using digital computers," IEEE Transactions on Power Delivery, vol. 3, no. 4, pp. 1511-1522, Oct 1988.

[5] P. M. Frank, "Fault diagnosis in dynamic systems using analytical and knowledge-based redundancy-a survey and some new results," Automatica, vol. 26, pp. 459-474, 1990.

[6] J. J. Gertler, Fault Detection and Diagnosis in Engineering Systems. Marcel Dekker, Inc., 1998.

[7] Hydro-Qubec and T. Technologies, SimPowerSystems 4 User's Guide, The MathWorks, Inc., 3 Apple Hill Drive Natick, MA 01760-2098, March 2008.

[8] R. Isermann, "Model-based fault-detection and diagnosis - status and applications," Annual Reviews in Control, vol. 29, pp. 71-85, 2005.

[9] P. C. Krause, O. Wasynczuk, and S. D. Sudhoff, Analysis of Electric Machinery and Drive Systems. Piscataway, NJ: IEEE Press Power Engineering Series, 2002.

[10] Simulink 7 Using Simulink, The MathWorks, Inc., 3 Apple Hill Drive Natick, MA 01760-2098, March 2008.

[11] A. Megahed and O. Malik, "An artificial neural network based digital differential protection scheme for synchronous generator stator winding protection," IEEE Transactions on Power Delivery, vol. 14, no. 1, pp. 86-93, Jan 1999.

[12] — , "Synchronous generator internal fault computation and experimental verification," IEE Proceedings-Generation, Transmission and Distribution, vol. 145, no. 5, pp. 604-610, Sep 1998.

[13] C.-M. Ong, Dynamic Simulation of Electric Machinery Using Matlab ${ }^{T M}$ / Simulink. Prentice-Hall, Inc., 1998.
[14] J. Penman and H. Jiang, "The detection of stator and rotor winding short circuits in synchronous generators by analysing excitation current harmonics," International Conference on Opportunities and Advances in International Electric Power Generation, pp. 137-142, 18-20 Mar 1996.

[15] M. Senesky, G. Eirea, and T. J. Koo, "Hybrid modeling and control of power electronics," in Hybrid Systems: Computation and Control, ser. Lecture Notes in Computer Science. Springer Berlin / Heidelberg, 2003, vol. 2623/2003, pp. 450-465.

[16] J. Sottile, F. Trutt, and A. Leedy, "Condition monitoring of brushless three-phase synchronous generators with stator winding or rotor circuit deterioration," IEEE Transactions on Industry Applications, vol. 42, no. 5, pp. 1209-1215, Sept.-Oct. 2006.

[17] L. Yonggang, Z. Hua, and L. Heming, "The new method on rotor winding inter turn short-circuit fault measure of turbine generator," IEEE International Conference on Electric Machines and Drives, IEMDC'03, vol. 3, pp. 1483-1487 vol.3, 1-4 June 2003.

Ashraf Tantawy received the B.Sc. in electronics and communication engineering from Ain Shams University, Cairo, Egypt, in 1995. He has received both an M.Sc. in computer science from the University of Louisville, KY, in 2005, and an M.Sc. in electrical engineering from Vanderbilt University, Nashville, TN, in 2008, where he is currently working toward his Ph.D. From 1995 to 2005, he was a telecommunication and control systems engineer working in the design and implementation of communication and control systems for the oil and gas industry. Since January 2006, he has been a Graduate Research Assistant with the Institute for Software Integrated Systems, Department of Electrical Engineering and Computer Science, Vanderbilt University, Nashville, TN. His research interests include detection and estimation theory, sensor networks, embedded systems and control, and modeling and simulation of complex hybrid systems.

Xenofon Koutsoukos is an Assistant Professor in the Department of Electrical Engineering and Computer Science at Vanderbilt University and a Senior Research Scientist in the Institute for Software Integrated Systems. His research interests include hybrid systems, real-time embedded systems, and sensor networks. He received the Diploma in electrical and computer engineering from the National Technical University of Athens, Athens, Greece, in 1993, M.S. degrees in electrical engineering and applied mathematics, and the Ph.D. degree in electrical engineering from the University of Notre Dame in 1998 and 2000, respectively. From 2000 to 2002, he was a member of Research Staff with the Xerox Palo Alto Research Center (PARC) working in the Embedded Collaborative Computing Area. He currently serves as Associate Editor for the ACM Transactions on Sensor Networks and for Modelling Simulation Practice and Theory. Dr. Koutsoukos is a senior member of IEEE and a member of ACM. He was the recipient of the National Science Foundation CAREER Award in 2004.

Gautam Biswas is a Professor of Computer Science and Computer Engineering in the EECS Department and a Senior Research Scientist at the Institute for Software Integrated Systems (ISIS) at Vanderbilt University. He has a Ph.D. degree in Computer Science from Michigan State University in E. Lansing, MI. His primary interests are in hybrid modeling, simulation, and analysis of complex embedded systems, and their applications to diagnosis and faultadaptive control. He has also worked on planning and scheduling algorithms for robotic task planning in uncertain environments, and dynamic resource allocation in distributed real-time environments. In other research projects, he is involved in developing simulation-based environments for learning and instruction. He has published extensively, and has over 300 publications. $\mathrm{He}$ is a senior member of the IEEE Computer Society, ACM, AAAI, and the Sigma Xi Research Society. 


\section{APPENDIX}

The following are expressions for the continuous time model matrices that are presented in Section III

$$
\begin{gathered}
L_{s}=\left[\begin{array}{ccc}
L_{l s}+L_{A}-L_{B} \cos 2 \theta_{r} & -\frac{1}{2} L_{A}-L_{B} \cos 2\left(\theta_{r}-\frac{\pi}{3}\right) & -\frac{1}{2} L_{A}-L_{B} \cos 2\left(\theta_{r}+\frac{\pi}{3}\right) \\
-\frac{1}{2} L_{A}-L_{B} \cos 2\left(\theta_{r}-\frac{\pi}{3}\right) & L_{l s}+L_{A}-L_{B} \cos 2\left(\theta_{r}-\frac{2 \pi}{3}\right) & -\frac{1}{2} L_{A}-L_{B} \cos 2\left(\theta_{r}+\pi\right) \\
-\frac{1}{2} L_{A}-L_{B} \cos 2\left(\theta_{r}+\frac{\pi}{3}\right) & -\frac{1}{2} L_{A}-L_{B} \cos 2\left(\theta_{r}+\pi\right) & L_{l s}+L_{A}-L_{B} \cos 2\left(\theta_{r}+\frac{2 \pi}{3}\right)
\end{array}\right] \\
L_{s r}=\left[\begin{array}{ccc}
L_{s k q} \cos \theta_{r} & L_{s f d} \sin \theta_{r} & L_{s k d} \sin \theta_{r} \\
L_{s k q} \cos \left(\theta_{r}-\frac{2 \pi}{3}\right) & L_{s f d} \sin \left(\theta_{r}-\frac{2 \pi}{3}\right) & L_{s k d} \sin \left(\theta_{r}-\frac{2 \pi}{3}\right) \\
L_{s k q} \cos \left(\theta_{r}+\frac{2 \pi}{3}\right) & L_{s f d} \sin \left(\theta_{r}+\frac{2 \pi}{3}\right) & L_{s k d} \sin \left(\theta_{r}+\frac{2 \pi}{3}\right)
\end{array}\right] \\
L_{r}=\left[\begin{array}{ccc}
L_{l k q}+L_{m k q} & 0 & 0 \\
0 & L_{l f d}+L_{m f d} & L_{f d k d} \\
0 & L_{f d k d} & L_{l k d}+L_{m k d}
\end{array}\right] \\
\mathbf{M}=\left[\begin{array}{cc}
M_{11} & M_{12}
\end{array}\right]
\end{gathered}
$$$$
M_{11}=\left[\begin{array}{ccc}
L_{l s}+\frac{3}{2} L_{A}-L_{B} \cos 2 \theta_{r}+L_{B} \cos 2\left(\theta_{r}-\frac{\pi}{3}\right) & -\frac{3}{2} L_{A}-L_{l s}-L_{B}\left(\cos 2\left(\theta_{r}-\frac{\pi}{3}\right)-\cos 2\left(\theta_{r}-\frac{2 \pi}{3}\right)\right) & L_{B}\left(\cos 2 \theta_{r}-\cos 2\left(\theta_{r}+\frac{\pi}{3}\right)\right) \\
1 & 1 & 0 \\
0 & 0 & 0 \\
-L_{s k q} \cos \theta_{r} & -L_{s k q} \cos \left(\theta_{r}-\frac{2 \pi}{3}\right) & -L_{s k q} \cos \left(\theta_{r}+\frac{2 \pi}{3}\right) \\
-L_{s f d} \sin \theta_{r} & -L_{s f d} \sin \left(\theta_{r}-\frac{2 \pi}{3}\right) & -L_{s f d} \sin \left(\theta_{r}+\frac{2 \pi}{3}\right) \\
-L_{s k d} \sin \theta_{r} & -L_{s k d} \sin \left(\theta_{r}-\frac{2 \pi}{3}\right) & -L_{s k d} \sin \left(\theta_{r}+\frac{2 \pi}{3}\right)
\end{array}\right]
$$$$
M_{12}=\left[\begin{array}{ccc}
-L_{s k q} \cos \theta_{r}+L_{s k q} \cos \left(\theta_{r}-\frac{2 \pi}{3}\right) & -L_{s f d} \sin \left(\theta_{r}\right)+L_{s f d} \sin \left(\theta_{r}-\frac{2 \pi}{3}\right) & -L_{s k d} \sin \left(\theta_{r}\right)+L_{s k d} \sin \left(\theta_{r}-\frac{2 \pi}{3}\right) \\
0 & 0 & 0 \\
0 & 0 & 0 \\
L_{l k q}+L_{m f d} & 0 & 0 \\
0 & L_{l f d}+L_{m f d} & L_{f d k d} \\
0 & L_{f d k d} & L_{l k d}+L_{m k d}
\end{array}\right]
$$$$
\mathbf{N}=\left[\begin{array}{ll}
N_{11} & N_{12}
\end{array}\right]
$$

$$
N_{11}=\left[\begin{array}{ccc}
-2 \omega L_{B}\left(\sin \left(2 \theta_{r}\right)-\sin \left(2 \theta_{r}-\frac{2 \pi}{3}\right)\right)-r_{a}-r_{b}-R & -2 \omega L_{B}\left(\sin \left(2 \theta_{r}-\frac{2 \pi}{3}\right)-\sin \left(2 \theta_{r}-\frac{4 \pi}{3}\right)\right) & -2 \omega L_{B}\left(\sin \left(2 \theta_{r}+\frac{2 \pi}{3}\right)-\sin \left(2 \theta_{r}\right)\right) \\
0 & 0 & 0 \\
0 & 0 & 0 \\
\omega L_{s k q} \sin \theta_{r} & \omega L_{s k q} \sin \left(\theta_{r}-\frac{2 \pi}{3}\right) & \omega L_{s k q} \sin \left(\theta_{r}+\frac{2 \pi}{3}\right) \\
-\omega L_{s f d} \cos \theta_{r} & -\omega L_{s f d} \cos \left(\theta_{r}-\frac{2 \pi}{3}\right) & -\omega L_{s f d} \cos \left(\theta_{r}+\frac{2 \pi}{3}\right) \\
-\omega L_{s k d} \cos \theta_{r} & -\omega L_{s k d} \cos \left(\theta_{r}-\frac{2 \pi}{3}\right) & -\omega L_{s k d} \cos \left(\theta_{r}+\frac{2 \pi}{3}\right)
\end{array}\right]
$$

$$
N_{12}=\left[\begin{array}{ccc}
-\omega L_{s k q} \sin \theta_{r}+\omega L_{s k q} \sin \left(\theta_{r}-\frac{2 \pi}{3}\right) & \omega L_{s f d} \cos \left(\theta_{r}\right)-\omega L_{s f d} \cos \left(\theta_{r}-\frac{2 \pi}{3}\right) & \omega L_{s k d} \cos \left(\theta_{r}\right)-\omega L_{s k d} \cos \left(\theta_{r}-\frac{2 \pi}{3}\right) \\
0 & 0 & 0 \\
0 & 0 & 0 \\
-r_{k q} & 0 & 0 \\
0 & -r_{f d} & -r_{k d}
\end{array}\right]
$$

